\title{
Complete loss of function of the ubiquitin ligase HERC2 causes a severe neurodevelopmental phenotype
}

\author{
Fanny Morice-Picard ${ }^{\star, 1,2}$, Giovanni Benard ${ }^{1}$, Hamid R Rezvani ${ }^{3}$, Eulalie Lasseaux ${ }^{2}$, Delphine Simon ${ }^{1}$, \\ Sébastien Moutton ${ }^{1}$, Caroline Rooryck ${ }^{1,2}$, Didier Lacombe ${ }^{1}$, Clarisse Baumann ${ }^{4}$ and Benoit Arveiler ${ }^{1,2}$
}

The ubiquitin-proteasome pathway is involved in the pathogenesis of several neurogenetic diseases. We describe a Mauritanian patient harboring a homozygous deletion restricted to two contiguous genes HERC2 and OCA2 and presenting with severe developmental abnormalities. The deletion causes the complete loss of HERC2 protein function, an E3-ubiquitin ligase. HERC2 is known to target XPA and BRCA1 for degradation and a mechanism whereby it is involved in DNA repair and cell cycle regulation. We showed that loss of HERC2 function leads to the accumulation of XPA and BRCA1 in the patient's fibroblasts and generates decreased sensitivity to apoptosis and increased level of DNA repair. Our data describe for the first time the phenotypic consequences, both at the clinical and cellular levels, of a complete loss of HERC2 function in a patient. They strongly suggest that profound ubiquitin ligase - associated dysfunction is responsible for the severe phenotype in this patient, and that dysfunction of this pathway may be involved in other patients with similar neurodevelopmental diseases.

European Journal of Human Genetics (2017) 25, 52-58; doi:10.1038/ejhg.2016.139; published online 19 October 2016

\section{INTRODUCTION}

HERC2 (HECT domain and RCC1-like domain 2, MIM 605837, NM_004667.4) is a pigmentation-unrelated gene located $11.7 \mathrm{~kb}$ upstream of OCA2. Mutations of the OCA2 gene are responsible for non syndromic oculocutaneous albinism type 2. OCA2 spans $345 \mathrm{~kb}$ in $15 \mathrm{q} 11.2-\mathrm{q} 12$ and contains 25 exons. It encodes the $\mathrm{P}$ protein which is a membrane component of the melanosome that might be involved in the regulation of melanosomal $\mathrm{pH}$, an essential factor in the various stages of melanin production and, in particular, in the relative rate of eumelanin and pheomelanin amounts. Deletions represent $14.5 \%$ of OCA2 mutations. ${ }^{1}$ Sequences homologous to the HERC2 gene constitute low-copy repeats associated with breakpoints of chromosomal rearrangements located in 15q11-15q13. ${ }^{2}$ HERC2 encodes a $528 \mathrm{kDa}$ E3 ubiquitin ligase, that interacts with E6AP/UBE3A, another ubiquitin ligase, and targets BRCA1 and XPA for degradation. ${ }^{3-5}$ Until recently, HERC2 mutations were not associated with a human disorder. Lately, a single homozygous mutation of HERC2 was found to segregate in Old Order Amish families with a neurodevelopmental disorder that has some phenotypic similarities to Angelman Syndrome. ${ }^{6,7}$

Here we present a previously undescribed phenotype in an African patient presenting with OCA and a severe neurological and developmental disorder associated with a large homozygous deletion involving the two contiguous genes OCA2 and HERC2.

\section{MATERIALS AND METHODS}

Array-CGH

A microarray was specifically designed to search for deletions in the OCA1-4 genes, as already described. ${ }^{1}$ The variant has been deposited in the LOVD HERC2 mutation database (http://www.LOVD.nl/HERC2). The Patient's ID given is 00072301 .
Sequencing of the deletion breakpoints

Primers used to amplify the deletion breakpoint in the patient were 5'GTAGCTACTAAAATGAATTGGACAAA3' and 5'ATGAACTTTATAAGCA GCAAAGCA3'. Both strands of the two PCR fragments were sequenced using the primers used for PCR amplification using the Big Dye v1.1 kit on an ABI3130xl sequencer (Applera, Courtaboeuf, France). PCR and sequencing cycling conditions are available from the authors upon request. Analysis of the sequence surrounding the deletion breakpoints was performed using softwares NCBI BLAST2 for sequence homology search and RepeatMasker for the detection of repetitive sequences (NCBI, BLAST2, http://www.ncbi.nlm.nih. gov/ blast/bl2seq/bl2.html; RepeatMasker, http://www.repeatmasker.org/). Accession Numbers of the HERC2 and OCA2 gene sequences used were RefSeq NG_016355.1 (NM_004667.4) and NG_009846.1 (NM_000275.2), respectively.

\section{RNA studies}

Total RNA was extracted from cells or tissues using Trizol. Real-time qPCR amplifications were performed as described previously. ${ }^{8}$ Succinctly, PCR reactions contained IX IQ SYBR Green Supermix (Biorad, Steenvoorde, France), the two primers required for amplification and either $50 \mathrm{ng}$ of total DNA or cDNAs from $100 \mathrm{ng}$ of reverse-transcribed total RNA. Sequences of the primers were as follows. Fragment HERC2-1 (spanning HERC2 exons 5 and 7): 5'CGGTATTTC TCTGCCTCCTG3', 5'ACTCCTGCAACAGCTCACTG3'. Fragment HERC2-2 (spanning HERC2 exons 90 and 91): 5'ACACACATCACCCTGGACAA3', 5'CC GGTGAACAGAGAGAGGAG3'. Fragment OCA2-23 (spanning OCA2 exons 23 and 24): 5'TGGATATGGGTTCTCCTTCA3', 5'ACACATCCCAACAGTGC AG3'. Fragment HERC2-OCA2 (spanning HERC2 exon 56 and OCA2 exon 20 in the HERC2-OCA2 fusion RNA): 5'ATCCATGGTCTCATCCTGCT3', 5'A CACATCCCAACAGTGCAG3'. The RPLP0 mRNA served as an internal control for normalization. PCR conditions are available from the authors on request.

${ }^{1}$ Univ. Bordeaux, Maladies Rares: Génétique et Métabolisme (MRGM) EA4576, Bordeaux, France; ${ }^{2} \mathrm{CHU}$ de Bordeaux, Service de Génétique Médicale, Bordeaux, France; ${ }^{3}$ INSERM, Biothérapies des Maladies Génétiques et Cancers, Bordeaux, France; ${ }^{4}$ Hôpital Robert Debré, Service de Génétique Médicale, Paris, France

*Correspondence: Dr F Morice-Picard, Laboratoire Maladies Rares - Génétique et Métabolisme (EA 4576) Ecole de Sages-Femmes Hôpital Pellegrin, 1 Place Amélie Raba-Léon, Bordeaux Cedex 33076, France. Tel: +33 5578203 55; Fax: +33 5567956 48; E-mail: fanny.morice-picard@chu-bordeaux.fr

Received 29 March 2016; revised 28 August 2016; accepted 13 September 2016; published online 19 October 2016 


\section{Protein studies}

Cell culture. Patient and control fibroblasts were maintained at $37^{\circ} \mathrm{C}$ and $5 \%$ $\mathrm{CO}_{2}$ in DMEM medium (Gibco, Life Technologies, Grand Island, NY, USA) supplemented with $10 \%$ fetal bovine serum and antibiotics. Cells were grown to $90-100 \%$ confluence in T75 cell culture flasks.

Immunocytochemistry. Cells were transferred to a four-well plate for fixation. Cells on coverslips were fixed for $30 \mathrm{~min}$ in $4 \%$ paraformaldehyde in PBS, permeabilized and blocked for 60 min with $0.3 \%$ Triton X-100 and 3\% normal goat serum in PBS, and incubated with specific antibodies for $1 \mathrm{hr}$ at room temperature (RT): monoclonal anti-HERC2, the epitope of which lies within the predicted HERC2-OCA2 fusion protein (aa 1781-1974; 1:500, BD Biosciences, San Jose, CA, USA); polyclonal anti-Ubi-Lys48 (1:500; Millipore, Temecula, CA, USA); monoclonal anti-BRCA1 (1:500; Abcam, Cambridge, UK). Following a wash in PBS, cells were incubated in AlexaFluor 488conjugated goat anti-mouse $\mathrm{IgG}_{1}$ and AlexaFluor 568-conjugated goat antirabbit (1:500; Life Technologies) for $1 \mathrm{hr}$ at RT. Coverslip were then washed 3 times five min with PBS, and mounted with Prolongold containing DAPI $\left(4^{\prime}, 6\right.$ oui-diamidino-2-phenylindole; Life Technologies). Images were acquired using an AxioVision microscope (Zeiss, Göttingen, Germany) with a $\times 63$ objective. Colocalization analyses were performed using the Intensity Correlation Analysis plug-in from the MacBiophotonics ImageJ software.

\section{Determination of 6-4PP and CPD repair kinetics}

Repair kinetics was investigated as previously described. ${ }^{9}$ Briefly, exponentially growing asynchronous fibroblasts were exposed to UVB at a dose of $50 \mathrm{~mJ} \mathrm{~cm}$ -2 using a Biotronic device (Vilber Lourmat, Marne la Vallée, France), equipped with a dosimeter, in which the UVB lamp emitted a continuous spectrum between 280 and $380 \mathrm{~nm}$ (major peak at $312 \mathrm{~nm}$ ). At different time intervals after irradiation, cells were fixed with $4 \%$ formaldehyde for $10 \mathrm{~min}$ at room temperature and then permeabilized overnight in cold 70\% ethanol. Cells were then resuspended in either $0.5 \%$ Triton-X-100/2 N HCl (for CPD detection) or $0.5 \%$ Triton-X-100/0.2 N HCl (for 6-4PP detection) for $10 \mathrm{~min}$ at room temperature. After washing with Tris-base $1 \mathrm{M}(\mathrm{pH} \mathrm{10)}$ and then with PBS, fibroblasts were incubated overnight at $4{ }^{\circ} \mathrm{C}$ with $100 \mathrm{ml}$ of PBS-TF $(4 \%$ FBS/0.25\% Tween-20/PBS) containing a 1:100 dilution of either the anti-6-4PP (Kamiya Biomedical company, Seattle,WA, USA) or anti-CPD (Kamiya Biomedical company) antibodies. After washing twice with PBS, cells were resuspended in $100 \mathrm{ml}$ PBS-TF containing Alexa- Fluor 488-coupled secondary antibody (1:200) for $1 \mathrm{~h}$ at room temperature. Repair kinetics was then monitored using flow cytometry (FACSalibur, Becton Dickinson, San Jose, CA, USA) to quantify the change in geometric mean fluorescence over time. Unpaired Student's $t$ test comparing the value between control and patient in each time was used on four independent studies.

\section{Apoptosis analysis}

Evaluation of UVB-induced apoptosis and necrosis was performed as previously described. ${ }^{9}$ Briefly, $16 \mathrm{~h}$ after irradiation, fibroblasts were incubated with $5 \mu \mathrm{mol} / \mathrm{l}$ FITC-VAD-FMK (Promega, Charbonnières, France) for $30 \mathrm{~min}$ at $37^{\circ} \mathrm{C}$ in the dark and then with $2.5 \mu \mathrm{g} / \mathrm{ml}$ propidium iodide (PI, Sigma, Saint Quentin Fallavier, France). Cells were immediately analyzed by flow cytometry.

To evaluate the activation of caspase-3/7, Caspase-Glo -3/7 assay (Promega Corp., Charbonnières, France) have been used. The assay provides a proluminescent caspase-3/7 that is cleaved to aminoluciferin. The released aminoluciferin is a substrate that is consumed by the luciferase, generating a luminescent signal. The signal is proportional to caspase- $3 / 7$ activity. After $16 \mathrm{~h}$ ultravioletB irradiation, fibroblasts were incubated with proluminescent caspase-3/7 substrate for $30 \mathrm{~min}$ at $37^{\circ} \mathrm{C}$ in the dark and then luminescent signal was measured by the GloMax luminometer (Promega).

\section{Statistics}

All values are the mean \pm SEM. The statistics were performed with the GraphPad Prism 5 software using an unpaired Student's $t$ test to compare two independent groups or pairs for sequential measurements. One-way and two-way ANOVA were performed when comparing different groups.

\section{RESULTS}

\section{Patient}

The patient is a new-born male who was the fourth child of healthy first-cousin Mauritanian parents. In the family history, the mother had a spontaneous abortion for unknown reasons at 28 weeks of gestation. The three older children were healthy. Family history revealed that one brother and one sister of the mother died early in infancy with albinism associated with an unknown disorder. Concerning the patient, antenatal ultrasound examination showed enlargement of pericerebellar spaces. The parents refused further prenatal investigations such as fetal MRI or amniocentesis. The patient was born at term after an unremarkable delivery with a weight, height and head circumference in standard ranges. He was presenting with a homogeneous hair and skin depigmentation evocative of oculocutaneous albinism (Figure 1a). Ophthalmologic examination showed moderate retinal hypopigmentation (not shown). At 4 months, he had a growth delay associated with feeding difficulties. Echocardiography revealed left heart dilatation, a large patent ductus arteriosus and pulmonary hypertension. Neurologic examination showed axial hypotonia, peripheral hypertonia associated with extrapyramidal symptoms and uncoordinated movements. Brain MRI confirmed the enlargement of pericerebellar spaces compatible with a diagnosis of megacisterna magna (Figures $1 \mathrm{~b}$ and $\mathrm{c}$ ), associated with bilateral perisylvian polymicrogyria (Figures 1c and e) and hypoplastic corpus callosum. At 14 months feeding difficulties led to gastrostomia. Recurrent pulmonary infections required hospitalization in intensive care. The patient died at 2 years of age after a pulmonary decompensation. Chromosomal analysis and genome-wide microarray (Agilent, $60 \mathrm{~K}$ ) did not reveal any anomaly (46XY). A diagnosis of oculocutaneous albinism associated with an unknown associated disorder was suggested.

\section{Genetic studies}

Because the patient's complex phenotype suggested the existence of a contiguous gene syndrome, a search for a deletion involving one of the OCA genes and surrounding regions was performed. For this purpose we used a high resolution comparative genomic hybridization (CGH) array covering the OCA1-4 genes and neighboring genomic segments with an average spacing between probes of $100 \mathrm{bp} .{ }^{1}$ We identified a homozygous $286 \mathrm{~kb}$ deletion extending between coordinates chr15:28143765 and chr15: 28429460 (Hg19) (chr15: g. 28143765_28429460 del) (Figure 2a). We PCR-amplified, using primers located in intron 56 of HERC2 and intron 19 of OCA2, a 581 bp fragment encompassing the deletion breakpoints (Figure $2 \mathrm{~b}(\mathrm{i}$ and ii)). Our data showed that the deletion fused exons 1-56 of HERC2 to exons 20-25 of OCA2. This suggested that the deletion of OCA2 accounts for the albino phenotype as previously described and that the additional severe clinical manifestations could be explained by the deletion of HERC2.

\section{Identification of a HERC2-OCA2 fusion transcript and absence of the HERC2 protein in patient cells}

We were able to amplify a fragment (OCA2-23) encompassing exons 23-24 of OCA2 from the patient's fibroblasts RNA, thus suggesting that the $3^{\prime}$ end of the OCA2 mRNA was expressed as part of a fusion HERC2-OCA2 transcript under the control of the HERC2 promoter. To further confirm that the fusion transcript was produced in the patient's cells, we amplified a 515 bp fragment encompassing HERC2 exon 56 and OCA2 exon 20 (fragment called HERC2-OCA2). Sequencing of this RT-PCR fragment showed that the fusion occurred at the expected position in the transcript. Altogether, these data demonstrated that a fusion transcript containing exons 1-56 of 

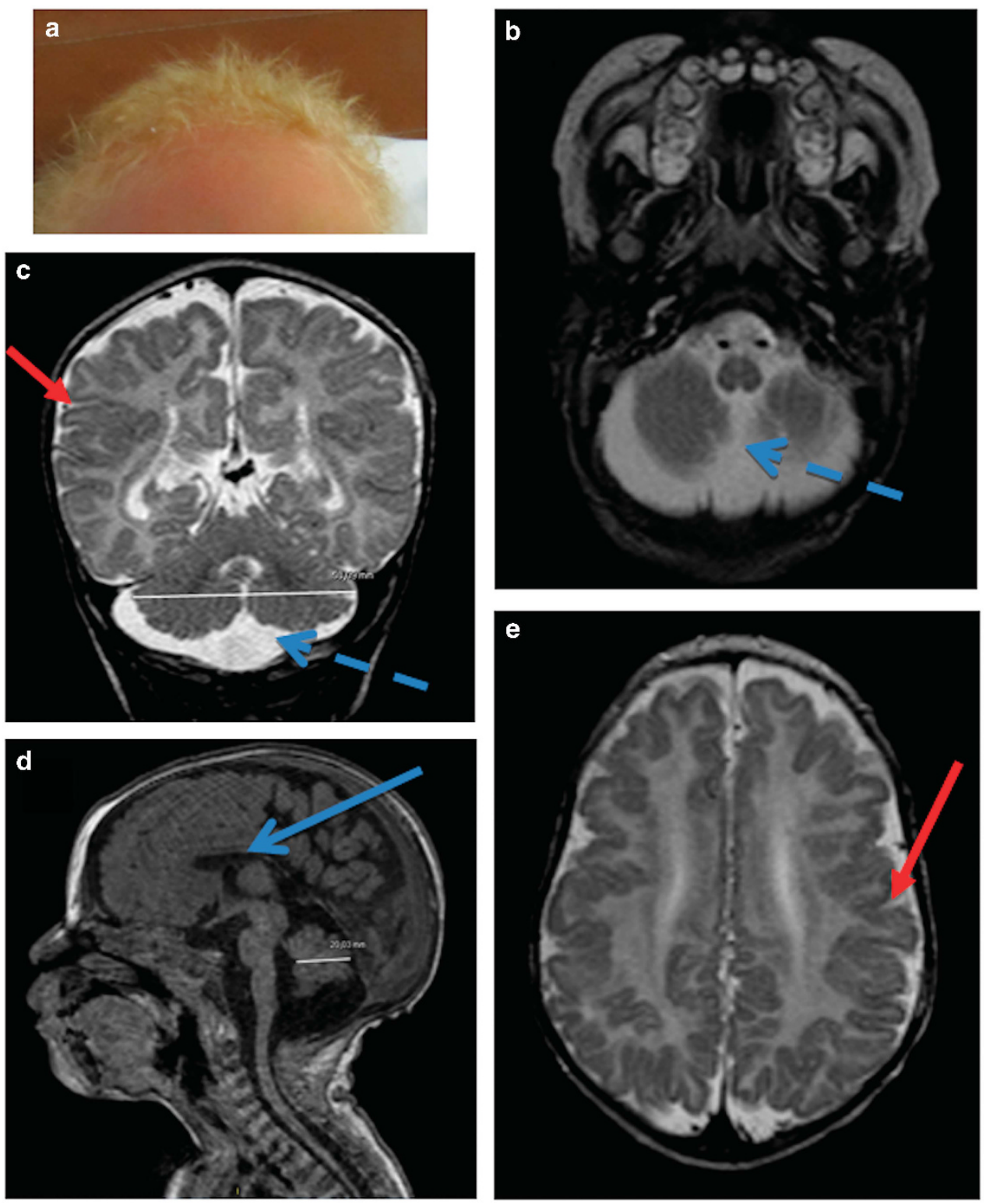

Figure 1 Patient's phenotype. (a) Hypopigmentation with blond hair and light skin. (b-e) MRI cross-sections; (b) T2 axial; (c) T2 coronal; (d) T1 sagittal; (e) T2 axial) showing enlargement of pericerebellar spaces (probably a mega cisterna magna; dashed blue arrow), irregular junction between white matter and gray matter and abnormality of gyration with polymicrogyria (red arrows) and hypoplasia of corpus callosum (blue arrow).

HERC2 followed by exons 20-25 of OCA2 was produced in the patient's fibroblasts.

In silico translation of this fusion transcript (software at http:// insilico.ehu.es/translate/) predicted the production of a truncated protein containing the first 2941 amino acids of HERC2 followed by 7 amino acids derived from the OCA2 sequence (out of frame) until a STOP codon occurred (Data not shown). The predicted fusion protein lacked the RCC1-like domains (RLD2, RLD3) (Regulator of Chromosome Condensation $=\mathrm{RCC} 1$ ) and the C-terminal Homologous to the E6-AP Carboxyl Terminus (HECT) domains (Figure 3a). By western blot analysis using a specific anti-HERC2 antibody the epitope of which lied within the predicted HERC2-OCA2 fusion protein we observed the absence of the HERC2 protein in the patient's fibroblasts compared with control, both at the wild-type HERC2 expected size
(Figure $3 \mathrm{~b}$ ) and at that of the predicted truncated protein. This suggested that the truncated protein was degraded.

Loss of function of HERC2 leads to ubiquitination anomalies and accumulation of its substrates

HERC2 is an E3-ubiquitin ligase involved in the ubiquitination of several substrates. ${ }^{3,5,10}$ Ubiquitination was studied in the patient's fibroblasts by immunocytochemistry using a specific anti-Ubi-Lys48 antibody. We observed an increased number of ubiquitin aggregates in the patient's cells compared with controls. $100 \%$ of the patient's cells contained aggregates, with an average number of 46 aggregates per cell $(S D=9,8)$. In contrast, only $40 \%$ of control's cells contained aggregates, with an average number of 5 aggregates per cell $(\mathrm{SD}=6,9)$, a difference that was statistically significant $(P<0,001$; Figures $3 \mathrm{c}$ and $\mathrm{d})$. 
a

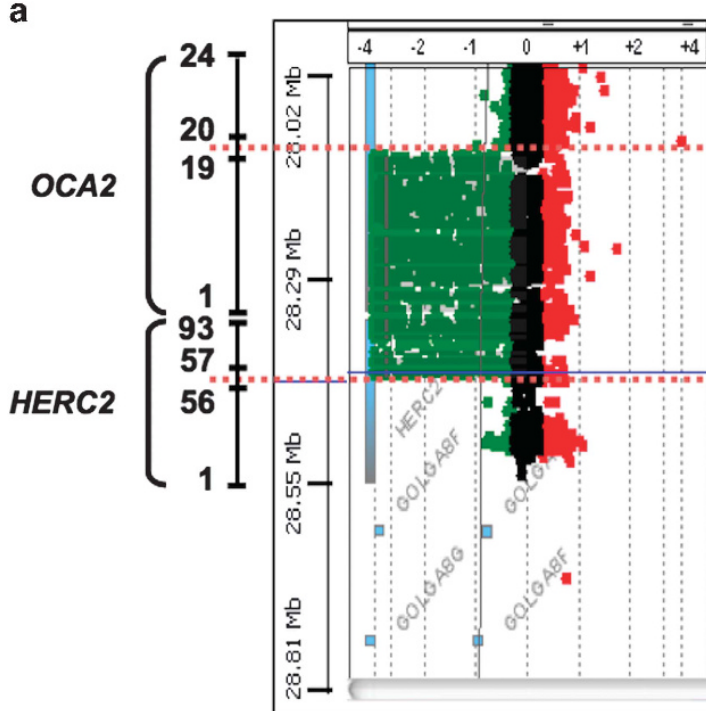

b

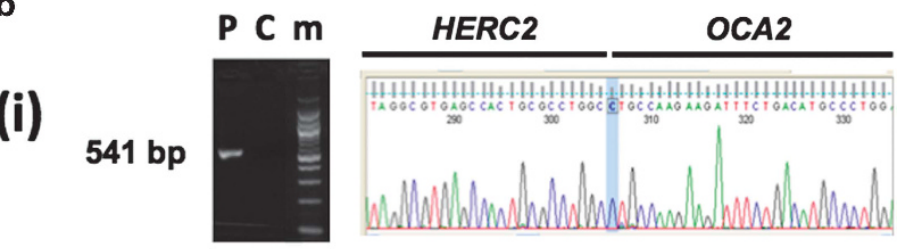

$\operatorname{chr} 15: 28429725$

(ii) Telomeric breakpoint (HERC2): 5' CAGCCTCCCAAAGTGCTGGGATTATAGGCGTGAGCCACTGCGCCTGGCIC]ATAAATTATAATAGATATAT

chr15: 28142054

Patient CAGCCTCCCAAAGTGCTGGGATTATAGGCGTGAGCCACTGCGCCTGGC[C]TGCCAAGAAGATTTCTGAC

Figure 2 Characterization of the DNA rearrangement. (a) Array-CGH analysis of the homozygous $285 \mathrm{~kb}$ HERC2-OCA2 deletion. Coordinates and Log2 values of the patient/control ratio of fluorescence are indicated on the left of and above the plot, respectively. The OCA2 and HERC2 genes are sketched on the left. Only key exons, represented by numbers, are shown. The dotted blue lines indicate the deletion breakpoints. (b) Sequencing of the HERC2-OCA2 deletion junction. (i) Amplification of a $541 \mathrm{bp}$ fragment containing the HERC2-OCA2 junction (P: patient; C: control individual not harboring the HERC2-OCA2 deletion; m: size marker). The electrophoregram displays the sequence including the HERC2-OCA2 junction. The cytosine at the junction is indicated (blue vertical stripe). (ii) Upper part: sequences surrounding the telomeric (HERC2) and centromeric (OCA2) deletion breakpoint. In blue: HERC2 unique sequences. In green $42 \mathrm{bp}$ repeat common to HERC2 and OCA2. The C between brackets corresponds to the cytosine highlighted in (a). In red: OCA2 unique sequences. Striped nucleotides correspond to deleted sequences. Lower part: sequence of the HERC2-OCA2 junction in the patient. Color codes are the same as in the upper part. Coordinates are according to the February 2009 Human reference sequence (NCBI Build 37.1/hg19).

HERC2 has been shown to target BRCA1 and XPA for degradation. ${ }^{3,5}$ It was expected that loss of the E3 ubiquitin ligase activity of HERC2 caused the accumulation of these proteins. In patient's cells, we observed deposits of BRCA1 in the cytosol (Supplementary Figure 1). A moderate accumulation of XPA was observed by western blotting in the patient's cells when compared with control $(P<0,01$; Supplementary Figure 1).

\section{Accumulation of HERC2 substrates involved in DNA repair}

We next investigated whether accumulation of the DNA repair factor XPA had an effect on the response of the patient's fibroblasts to ultravioletB irradiation. To this end, fibroblasts were UVB-irradiated and apoptosis was measured $16 \mathrm{~h}$ after irradiation by flow cytometry using benzyloxycarbonyl-valine-alanine-aspartic acid-fluoromethyl ketone (VAD-FMK), which binds to activated caspases, and propidium iodide (PI), as an indicator of plasma membrane permeability. The percentages of apoptotic $\left(\mathrm{VAD}-\mathrm{FMK}^{+} / \mathrm{PI}^{-}\right)$and late apoptotic plus necrotic cells $\left(\mathrm{VAD}-\mathrm{FMK}^{+} / \mathrm{PI}^{+}\right)$showed that the patient's cells had a $47 \%$ decrease of the sensitivity to UVB-induced apoptosis compared with control cells (Figure 4). Accordingly, measurement of ultravioletB-induced caspase activation revealed a clear reduction in the activation of caspase $3 / 7$ in the patient's fibroblasts compared with controls (Figure 4).

To further investigate the consequences of the accumulation of nucleotide excision repair (NER) factor XPA, we evaluated the repair kinetics of 6-4 photoproducts (6-4PPs) and cyclobutane pyrimidine dimers (CPDs), which are the most frequent types of photolesions removed primarily by NER with different kinetics. ${ }^{11,16}$ After exposure to UVB, there was a rapid drop of $6-4 \mathrm{PPs}$ resulting in $70 \pm 7 \%$ and $72 \pm 8 \%$ removal of $6-4 \mathrm{PPs} 2.5 \mathrm{~h}$ post-irradiation in control and patient fibroblast, respectively (Figure $4 \mathrm{~b}$ ). The gradual repair of $6-4 \mathrm{PPs}$ in the next hours led to $93 \pm 5 \%$ and $94 \pm 6 \%$ removal by $20 \mathrm{~h}$ in control and patient fibroblasts, respectively (Figure $4 \mathrm{~b}$ ). Kinetics of CPDs repair in irradiated control fibroblasts revealed that CPDs were gradually removed following UVB irradiation, that is $12 \pm 6 \%$ removal at $0.5 \mathrm{~h}$ after irradiation reaching slowly $53 \pm 6 \%$ and $64 \pm 5 \%$ at $10 \mathrm{~h}$ and $20 \mathrm{~h}$ after irradiation, respectively Figure $4 \mathrm{~b}$ ). In patient's fibroblasts, the immediate CPDs removal was approximately the same $(8 \% \pm 7$ by $0.5 \mathrm{~h})$ as in control cells, while the rate of late CPDs repair was higher than in the control, since $70 \pm 6 \%$ and $80 \pm 6 \%$ of CPD were removed at $10 \mathrm{~h}$ and $20 \mathrm{~h}$ post-irradiation, respectively (Figure $4 \mathrm{~b}$ ). The differences between two groups are significant at 5,10 and $20 \mathrm{~h}$ after irradiation with p values of $0.017,0.0067$ and 0.0058 , respectively. In a previous study, knockdown of HERC2 by siRNA was also shown to increase the steady-state level of XPA and the rate of repair of both ultraviolet photoproducts. ${ }^{3}$

Altogether, these results indicated that CPD repair was higher in the patient's cells compared with controls, whereas no significant difference was observed concerning 6-4PP repair. These anomalies were likely due to HERC2, not OCA2, loss of function. 
a

HERC2 Protein 4834 aa

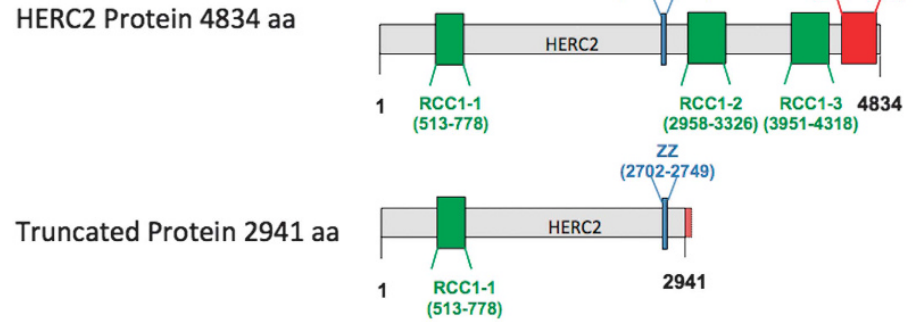

b

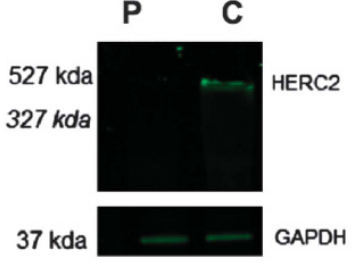

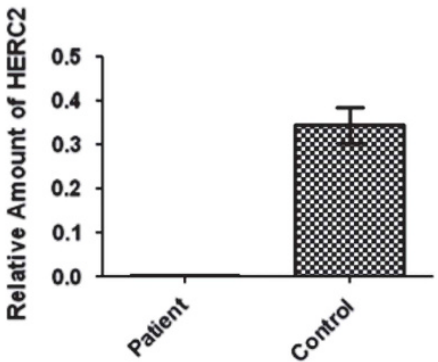

\section{C}

C
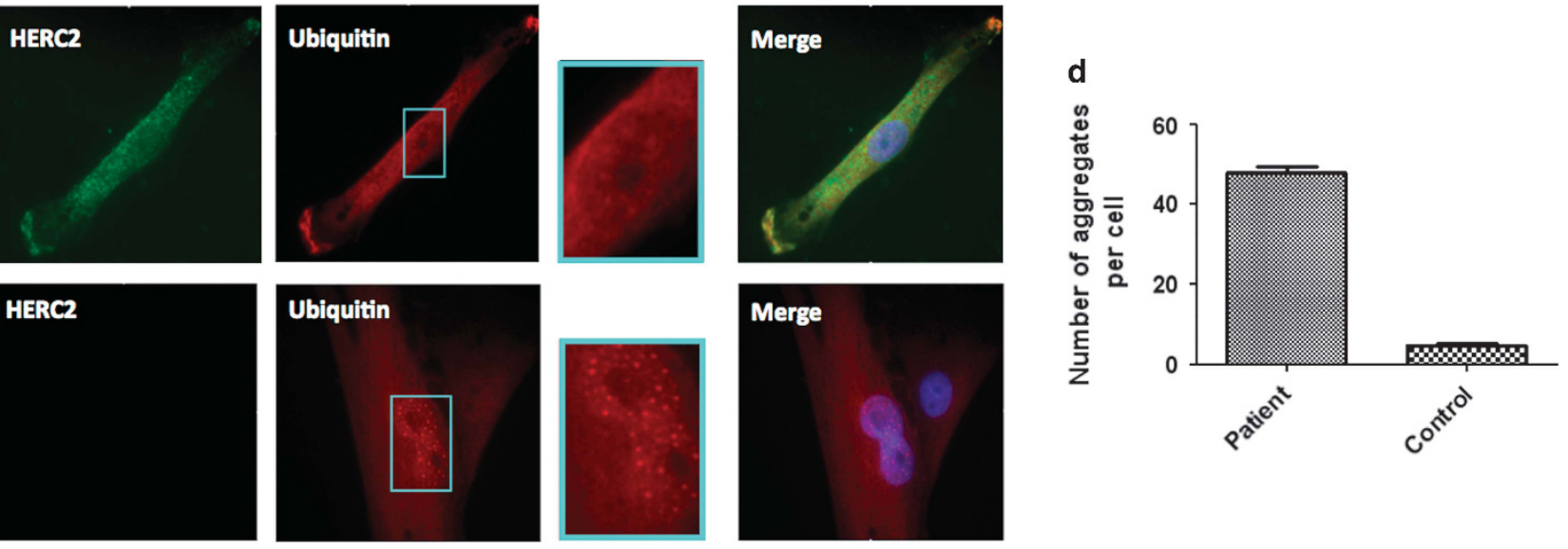

Figure 3 Loss of function of HERC2 causes the accumulation of ubiquitin aggregates. (a) Schematic representation of the HERC2 protein (upper) and HERC2-OCA2 predicted fusion protein (lower). The predicted fusion protein lacks 2 RCC1 (regulator of chromosome condensation) domains and the functional C-terminal HECT domain (Homologous to the E6-AP Carboxyl Terminus). (b) Western blot analysis of the patient's fibroblasts using an anti-HERC2 antibody the epitope of which lies within the predicted HERC2-OCA2 fusion protein (mean \pm SD of 3 independent experiments). P, patient; C, control. Expected sizes of wild type HERC2 and predicted HERC2-OCA2 fusion protein are $527 \mathrm{kDa}$ and $327 \mathrm{kDa}$ respectively (c) Control (C) and Patient (P) cells were incubated with anti-HERC2 (green) and anti-Ubiquitin (red) antibodies. Nuclei were stained with DAPI (blue). A representative cell is shown for the patient and the control. Intranuclear ubiquitin foci were detected in patient's cells (see red spots in magnified view of the nucleus on the right), when compared with the control. (d) Quantification of the number of ubiquitin aggregates per cell in the patient vs control (mean $\pm \mathrm{SD}$ of 50 cells; $P<0.001)$.

\section{DISCUSSION}

We identified a homozygous $286 \mathrm{~kb}$-large deletion encompassing exons 57 to 93 of the HERC2 gene and exons 1 to 19 of the OCA2 gene in a patient presenting with OCA and severe neurological anomalies.

The HERC2 protein belongs to the HERC protein family and shares with HERC1 its large size, RLD-1 domain at the N-terminus, and RLD and HECT domains at the C-terminus. ${ }^{12}$ Until recently the human HERC2 gene had not been causally associated with any human disease.

Rjs mice were reported to have neuromuscular and spermatogenic abnormalities and were characterized by defects in growth, movement coordination (jerky gait) and fertility. One of the rjs mutants expressed a truncated HERC2 protein lacking part of the C-terminal HECT domain. ${ }^{13}$ This indicated that loss of HERC2 E3 activity may be responsible for the observed phenotype in mice. A missense variant, (c.1781C > T, p.(Pro594Leu)), of the HERC2 gene was recently associated with an Angelman-like disorder in seven patients from the Old Order Amish community. 6,7 The phenotype observed in these patients included neonatal hypotonia, global developmental delay and gait instability. Cranial magnetic resonance imaging studies was normal for one patient and showed a mild cerebral atrophy normal in one additional. ${ }^{7}$ Clinical presentations of the seven patients were less severe than that of our patient, presumably because the mutated
HERC2 protein conserved part of its activity. The homozygous deletion identified in our patient results in a complete absence of HERC2 protein that likely explains the severity of the developmental abnormalities.

Interestingly, a homozygous nonsense mutation (c.9748C $>\mathrm{T}$, p. $\left.\left(\mathrm{R} 3250^{*}\right)\right)$ in another HERC gene, HERC1, was recently reported in a patient born to consanguineous parents and presenting with severe intellectual disability, megalencephaly, thick corpus callosum and a small cerebellum. ${ }^{14}$

Anomalies secondary to HERC2 loss of function may involve HERC2 substrates such as XPA and BRCA1, proteins that are ubiquitinated by HERC2. ${ }^{3,5}$ We have observed that a defect of HERC2 E3 ubiquitin ligase activity increased the abundance of XPA and BRCA1. Increased XPA and BRCA1 levels may affect DNA repair including DNA Double Strand (DDS) and Nucleotide Excision Repair (NER). Indeed we observed increased DNA repair of CPD photolesions and decreased sensitivity to apoptosis and caspase activity after UVB irradiation in the patient's cells. As DNA repair is a tightly regulated process, increased DNA repair and decreased sensitivity to apoptosis may have consequences on normal embryonic or fetal development, and may therefore participate in the phenotype observed in our patient. ${ }^{15}$ It was suggested that correct DNA repair is required for maintenance of the functional integrity of the nervous system by 


\section{口 C $\mathbf{P}$}

a
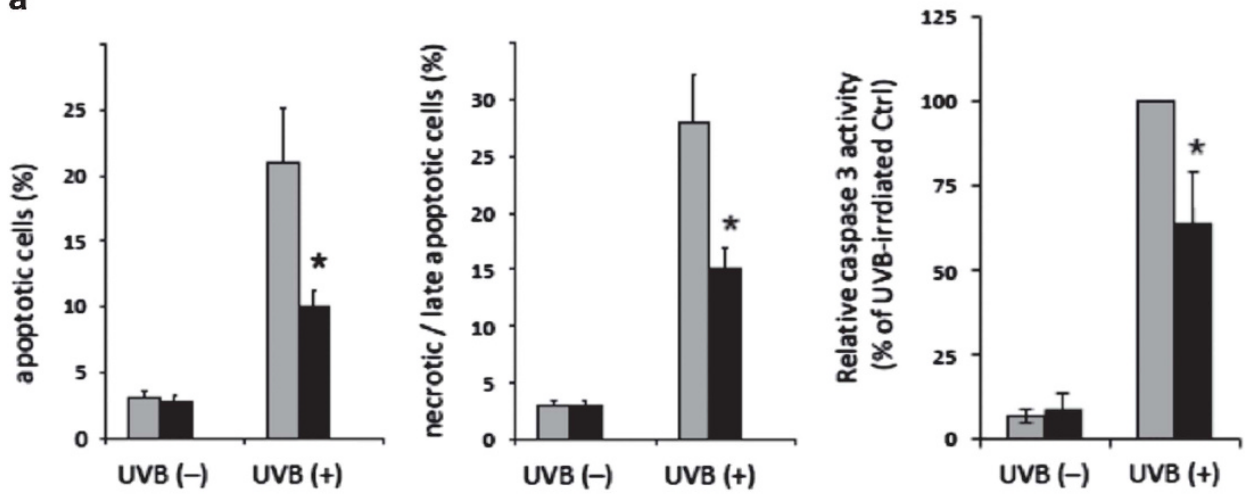

b
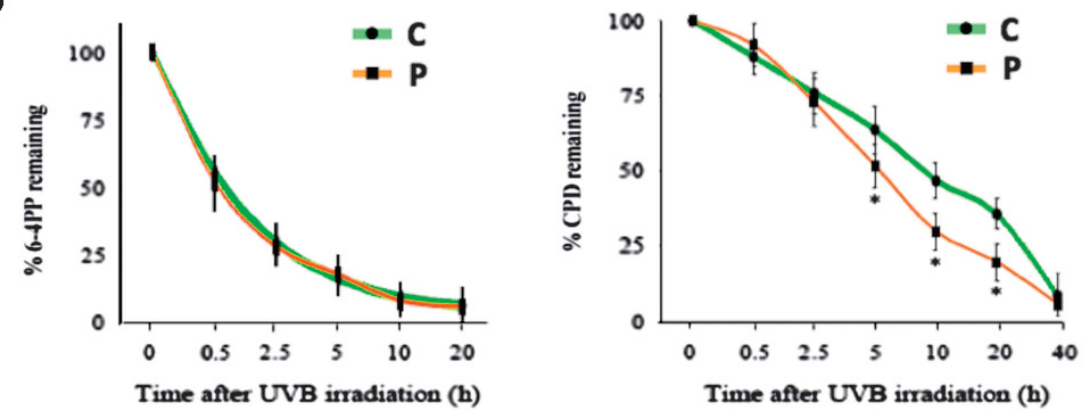

Figure 4 Accumulation of HERC2 substrates involved in DNA repair. (a) Analysis of apoptotic and late apoptotic/necrotic cells and of caspase-3 activity following UVB-irradiation. (b) Analysis of the repair kinetics of 6-4PPs (6-4 photoproducts) and CPD (cyclobutane pyrimidine dimers) after UVB irradiation. Less sensitivity to apoptosis is observed in patient's cells with slower relative caspase activity and increased rate of removal of CDP photolesions. No difference was observed upon 6,4PP removal. Results are expressed as the mean \pm SD of 4 independent experiments.

preventing the premature death of neurons. ${ }^{11}$ Further studies are needed to explain the pathophysiological link between these phenomenons.

HERC2 and UBE3A/E6AP, the loss of function of which causes Angelman syndrome, are ubiquitin ligases. HERC2 stimulates the ubiquitin-ligase activity of UBE3A/E6AP through its RLD2 domain. ${ }^{4}$ An impairment of UBE3A/E6AP E3 ligase activity due to a defect of HERC2 is expected to result in a decreased ubiquitination and degradation of UBE3A/E6AP substrates. The impact of HERC2 on UBE3A/E6AP and other specific substrates may contribute to the severity of the disease observed in the patient described here. ${ }^{10}$

The description of our patient with severe neurodevelopmental abnormalities who has a total loss of HERC2 function suggests that a profound ubiquitin ligase - associated dysfunction is responsible for abnormal brain development. HERC2 deletion could be associated to a more severe phenotype than missense mutation that was previously associated to autistic global developmental delay and autism spectrum disorder. ${ }^{6,7}$ This report expands the spectrum of HERC2-related disease. Further studies are required to understand the link between ubiquitination defects and neurodevelopmental abnormalities.

\section{CONFLICT OF INTEREST}

The authors declare no conflict of interest.

\section{ACKNOWLEDGEMENTS}

The authors are grateful to the patient and his family for their participation in this study. This work was supported by Genespoir (French Albinism Association) by the Ministry of Health and by the Ministry of Research of France. Funding: This work was supported by Genespoir (French Albinism Association), by Union Nationale des Aveugles et Déficients Visuels (France), by the Ministry of Health and by the Ministry of Research of France. FMP had a PhD studentship from the Fondation pour la Recherche Médicale (France). Sanger DNA Sequencing was performed at the Genome Transcriptome Platform of Bordeaux (grants from the Conseil Régional d'Aquitaine no 20030304002FA and 20040305003FA and from the European Union, FEDER no 2003227).

\footnotetext{
1 Morice-Picard F, Lasseaux E, Cailley D et al: High-resolution array-CGH in patients with oculocutaneous albinism identifies new deletions of the TYR, OCA2, and SLC45A2 genes and a complex rearrangement of the OCA2 gene. Pigment Cell Melanoma Res 2014; 27: 59-71.

2 Ji Y, Rebert NA, Joslin JM, Higgins MJ, Schultz RA, Nicholls RD: Structure of the highly conserved HERC2 gene and of multiple partially duplicated paralogs in human. Genome Res 2000; 10: 319-329.

3 Kang T-H, Reardon JT, Sancar A: Regulation of nucleotide excision repair activity by transcriptional and post-transcriptional control of the XPA protein. Nucleic Acids Res 2011; 39: 3176-3187.

4 Kühnle S, Kogel U, Glockzin S et al: Physical and functional interaction of the HECT ubiquitin-protein ligases E6AP and HERC2. J Biol Chem 2011; 286: 19410-19416.

$5 \mathrm{Wu}$ W, Sato K, Koike A et al: HERC2 is an E3 ligase that targets BRCA1 for degradation. Cancer Res 2010; 70: 6384-6392.
} 
6 Harlalka GV, Baple EL, Cross H et al: Mutation of HERC2 causes developmental delay with Angelman-like features. J Med Genet 2013; 50: 65-73.

7 Puffenberger EG, Jinks RN, Wang $\mathrm{H}$ et al: A homozygous missense mutation in HERC2 associated with global developmental delay and autism spectrum disorder. Hum Mutat 2012; 33: 1639-1646.

8 Rezvani HR, Dedieu S, North S et al: Hypoxia-inducible factor-1alpha, a key factor in the keratinocyte response to UVB exposure. J Biol Chem 2007; 282: 16413-16422.

9 Rezvani HR, Mahfouf W, Ali N et al: Hypoxia-inducible factor-1alpha regulates the expression of nucleotide excision repair proteins in keratinocytes. Nucleic Acids Res 2010; 38: 797-809.

10 Galligan JT, Martinez-Noël G, Arndt V et al: Proteomic analysis and identification of cellular interactors of the giant ubiquitin ligase HERC2. J Proteome Res 2015; 14: 953-966.
11 Brooks PJ, Cheng T-F, Cooper L: Do all of the neurologic diseases in patients with DNA repair gene mutations result from the accumulation of DNA damage? DNA Repair (Amst) 2008; 7: 834-848.

12 Garcia-Gonzalo FR, Rosa JL: The HERC proteins: functional and evolutionary insights. Cell Mol Life Sci 2005; 62: 1826-1838.

13 Lehman AL, Nakatsu Y, Ching A et al: A very large protein with diverse functional motifs is deficient in rjs (runty, jerky, sterile) mice. Proc Natl Acad Sci USA 1998; 95: 9436-9441.

14 Nguyen LS, Schneider T, Rio M et al: Mutation in HERC1 is associated with intellectual disability, megalencephaly, thick corpus callosum and cerebellar atrophy. Eur J Hum Genet 2016; 24: 455-458.

15 Mitchell DL: The relative cytotoxicity of (6-4) photoproducts and cyclobutane dimers in mammalian cells. Photochem Photobiol 1988; 48: 51-57.

Supplementary Information accompanies this paper on European Journal of Human Genetics website (http://www.nature.com/ejhg) 